Article

\title{
Modelling and Optimization in Resource Sharing Systems: Application to Bike Sharing with Unequal Demands
}

\author{
Xiaoting Mo ${ }^{1}{ }^{\mathbb{D}}$, Xinglu Liu ${ }^{1}$ and Wai Kin (Victor) Chan ${ }^{1, *}$ \\ 1 Intelligent Transportation and Logistics Systems Laboratory, Tsinghua-Berkeley Shenzhen Institute, \\ Shenzhen 518055, China; mxt17@tsinghua.org.cn; liuxl18@mails.tsinghua.edu.cn; chanw@sz.tsinghua.edu.cn \\ * Correspondence: chanw@sz.tsinghua.edu.cn
}

\begin{abstract}
Although the dockless bike-sharing system, which can be regarded as a typical example of the resource-sharing system, has been increasingly popular for years with people especially in China, the imbalanced distribution of shared bikes gradually becomes a major problem for both bike-sharing companies and their customers. To solve the imbalance problem, we aim to investigate the long-term performance of a system under the influence of some key factors (with an emphasis on the unequal demand between different nodes), which can guide us to discover the causes of the problem and offer several valuable suggestions to the operators. According to the fundamental principle of a dockless bike-sharing system, we propose a model reduction method to reduce the complexity of the theoretical network models, which are developed based on the Markovian queueing theory with the consideration of higher-demand nodes and lower-demand nodes. The theoretical network models provide us with steady-state probabilities of having a certain number of bikes at one node, which are used as an important part of the optimization model for solving the imbalance problem by carrying out an operator-based relocation strategy. The objective of the optimization model is to maximize the total profit and determine the optimal relocation frequency. It is found that most of the shared bikes are possible to gather at one low-demand node eventually in the long run under the influence of the different arrival rates at different nodes, but the decrease of the number of bikes at the high-demand nodes is more sensitive to the unequal demands and may cause a great loss for operators, which should be payed attention to especially when solving the relocation problems.
\end{abstract}

Keywords: dockless bike-sharing system; Markovian queueing network; relocation; unequal demand

\section{Introduction}

The existence of idle resources and people's willingness to make good use of them promote the development of the sharing economy, which brings several changes in lifestyle, including different traffic modes. The sharing economy means people can share resources (e.g., services, skills, or assets) through a network of private individuals and businesses, which may often but not always be at lower costs. Within the realm of resource sharing, shared transport has become more popular and common, such as ride sharing, car sharing, electric vehicle sharing and bike sharing. Obviously, the advent of the shared transport contributes to protecting the environment, conserving energy, reducing traffic congestion and improving utilization. But the present shared transport systems are not perfect. Sometimes users of these systems cannot find any available shared vehicle or bicycle in their area, or they have to spend too much time looking for it in the neighborhood, when they are in a hurry to leave. Sometimes vehicles or bikes are idle in several places where there is not enough demand from customers, making less profits for operators or drivers. There are many possible reasons, 
including the unpredictable demand, defective design and inappropriate operation. In essence, the phenomenon is caused by the imbalance between supply and demand. Hence, this paper focuses on bike sharing and is aimed at solving problems existing in bike-sharing systems. In addition, bike-sharing systems have two types: traditional bike-sharing system with docking stations and dockless bike-sharing system. The dockless bike-sharing system, as the main object of our study and the latest type of bike-sharing systems, is also called the free-floating bike-sharing system, which means users are allowed to park their bikes everywhere they want with no limitation of docking stations. As the dockless bike-sharing system appears, the traditional bike-sharing system begins to lose competitiveness and vanish gradually.

Initially, few studies talk about the feasibility and impacts of bike sharing as a new public transportation mode in urban areas ([1], [2], [3]). Recently, more papers begin to discuss the sustainable development of bike-sharing systems, especially the dockless bike-sharing systems. Because the study of dockless bike sharing is still in progress, and some mature studies on the station-based bike sharing can guide the development of the study on dockless bike sharing, we review literatures of research on both station-based bike sharing and dockless bike sharing. Some other typical transport sharing systems are mentioned due to their contributions to resource sharing. These literatures can be classified into three main parts: system design, system analysis and system optimization. Each part includes a review of some major topics and the relevant progress.

\subsection{System Design}

To build or expand a bike-sharing system, bike-sharing firms need to select an area and investigate the potential demand for shared bikes. Based on the essential research, these firms decide the scale of the network system, select bike pick-up locations, and allocate shared bikes to each node in the network to meet the potential demand. There are several ways to estimate demand by connecting with various factors. One traditional way is to do a population study and a sample survey to determine the locations of the pick-up/drop-off nodes in the system [4]. Frade and Ribeiro propose a methodology considering the distance and slope of city paths between any two traffic zones [5]. In the age of big data, some researchers extract valuable information from a large amount of historical data collected from existing bike-sharing systems. Xu et al. use deep learning approach with trip data to estimate dynamic demand for a citywide dockless bike-sharing system [6]. Besides demand prediction, how to build a bike-sharing network and where to allocate a number of bikes to serve users in the network are concerned. Çelebi et al. consider station locations and bike allocation using a set-covering model and a queueing model for a station-based bike-sharing system, given a number of stations [7].

\subsection{System Analysis}

In the operation phase of bike-sharing systems, a large amount of data can be collected and analyzed to find patterns (e.g., bike usage, trip characteristics) and make changes from operators' perspective. Bordagaray et al. use binary probit models to investigate travel behavior and impacts of different usage types (e.g., round trips, rental time reset, bike substitution) [8]. Gurumurthy et al. match different single-person trips by identifying similar time and routes from cellphone-based real-time data using MATLAB [9]. Yang et al. use dockless bike data to analyze bike mobility patterns based on unique bike IDs, including spatial and temporal patterns [10]. Ji et al. compare regularity of bike usage between station-based bike sharing and dockless bike sharing [11]. Besides usage pattern analysis, Bakogiannis et al. pay attention to user perceptions and evaluate the information to get ideas about how to improve user experience [12]. For operators, gaining profits is important. Profits have a close relationship with costs and revenues. Yoon et al. investigate the impacts of different pricing plans in several cities on membership demand and ridership, and present a new pricing plan based on the estimated cost per trip and price sensitivity of customers to improve revenue [13]. Estrada et al. focus on how to determine operational cost based on defined performance, expected functionality of a 
system and cost driver analysis [14]. Chen et al. aim at maximizing the profits by using the advantage of hassle costs, which are derived from the provided convenience of travel for customers [15].

\subsection{System Optimization}

Optimizing resource-sharing systems by relocation, which means relocating shared bikes to solve the problem of imbalanced distribution of bikes and try to make supply meet demand, is mainly discussed. A main principle is to move extra bikes from locations which have a surplus supply of bikes to locations which have a shortage of bikes. Relocation strategies have two primary types: operator-based relocation strategy and user-based relocation strategy [16]. An operator-based relocation strategy means relocation is driven by operators' behavior, and a user-based relocation strategy means relocation is directly driven by users, although all of the relocation strategies are operators' motivation and can benefit operators essentially. Studies about bike-sharing systems mainly focus on static and operator-based relocation problems. Sayar proposes a multi-periodic optimization formulation to get the minimum bike fleet size by maximizing the total profits [17]. Liu et al. solve a static relocation problem and minimize the weighted sum of three factors (i.e., inconvenience level of finding an available bike, lost demand and operational time) [18]. Brinkmann et al. propose a stochastic-dynamic lookahead policy to cope with changing demand patterns [19]. Legros uses a Markov decision process to determine the priority of stations where bikes need to be relocated and minimizes the ratio of arrival rate of unsatisfied users who cannot find any bikes to determine the number of relocated bikes at appointed stations [20]. Brendel et al. adopt a user-based relocation strategy to increase usage in an electric vehicle sharing system [21]. Reiss et al. combine operator-based relocation with user-based relocation, which is called as a hybrid relocation strategy, in a dockless bike-sharing system [22]. In an early stage, user-based relocation strategy may be sufficient to solve imbalance for the system. When user-based relocation is insufficient, operator-based relocation is applied by using trucks to relocate bikes through an effective route.

A minority of studies use queueing theory to model bike-sharing problems. Sayarshad et al. obtain the queueing delay by using the queueing-based approximation and relate the delay to a cost constraint of a dynamic relocation optimization model [23]. Samet et al. present a closed queu(e)ing network model for a station-based bike-sharing system [24]. For station-based bike-sharing systems, customers may wait at a station to pick up or return a bike, because the parking spaces are fixed and finite. For dockless bike-sharing systems, wait time is negligible. If customers can find an available bike to ride, they will not wait behind others. If customers cannot find one, they will leave quickly and feel dissatisfied.

The rest of this paper is organized as follows. In Section 2 we formulate the dockless bike-sharing system as a mathematical problem and describe how to build the theoretical network models based on the mentioned methods in general, which include the model reduction method and the method of Markovian queueing network. In Section 3 an operator-based relocation strategy is proposed to build an optimization model for solving relocation problems with the consideration of maximizing the total profit and finding out the optimal solutions for relocation frequency, which can be regarded as a decision-support tool for the decision-makers from the bike-sharing companies. In Section 4, we give an analysis and discussion of the steady-state probabilities derived from the theoretical models under the influence of unequal demands, and a profit analysis is provided with the change of the profit-related parameters based on the results from the optimization model. According to the researches in the previous sections, some valuable conclusions and suggestions about the dockless bike-sharing system, especially from the perspective of the operators, are presented in Section 5. 


\section{Markovian Queueing Networks}

\subsection{Assumptions and Notations}

The dockless bike-sharing system is modelled by a closed Markovian queueing network with $N \in \mathbb{N}^{*}$ connected nodes and a total of $K \in \mathbb{N}^{*}$ bikes. The bikes are distributed among the nodes $\mathcal{N}=\{1,2, \cdots, N\}$ and $\mathcal{K}=\{1,2, \cdots, K\}$ are the sets of $N$ nodes and $K$ bikes separately. Customers arrive at Node $i \in N$ to pick up bikes to ride and return bikes at Node $j \in N$. Let $p_{i j}$ be the transition probability from Node $i \in N$ to Node $j \in N$. All of the nodes are fully connected because of the customers' bike trips. The transition probabilities can reflect the information of customers' destinations as well as geographical environment. If the route between two nodes are long and steep, there will be a low probability of riding bikes between these two nodes. High demand leads to a large value of the arrival rate. The main assumptions are made as follows:

1. customers arrive at a node one by one for picking up bikes rather than arrive in groups;

2. the interarrival times of customers are exponentially distributed (i.e., the number of customer arrivals within a unit time interval is Poisson distributed) with an arrival rate $\lambda_{i}$ at Node $i$, and the arrivals at each node are completely independent;

3. all of the probabilities are the same for each route from a start node to a destination node (which can also be the start node itself), which means $p_{i j}=\frac{1}{N}$ for every pair of $i$ and $j$;

4. the time spent on picking up or dropping off bikes is negligible, which means customers do not have to wait in lines at any node in the network, and the trip time is not considered as an independent parameter in the theoretical models for what we care about is the steady states in the long run but is included in the simulation model;

5. the number of bikes is evenly distributed at each node at the beginning of the operation, and the total number of bikes in the system is fixed;

6. the capacity of each node is large enough to accommodate $K$ bikes;

7. if a node has no bike during a period of time, customers will still arrive randomly and leave immediately, and the service for these customers will be regarded as the lost demand.

And the notations are summarized in Table (1).

Table 1. Overview of notations.

\begin{tabular}{|c|c|c|}
\hline Notation & Units & Definitions \\
\hline$N$ & [nodes] & Total number of nodes in a system \\
\hline K & [bikes] & Total number of bikes in a system $(K=\epsilon N)$ \\
\hline$\epsilon$ & [bikes] & The initial number of bikes at each node in the system \\
\hline$\lambda_{i}$ & [people/min] & Arrival rate at Node $i \in N$ \\
\hline$\lambda$ & [people/min] & A certain value of the arrival rate \\
\hline$\alpha_{(m)}$ & [people/min] & $\begin{array}{l}\text { Transition rate of returning one bike to Node } i \text { by a } \\
\text { customer riding from one of the other nodes except Node } i \\
(m=0,1, \cdots, K-1)\end{array}$ \\
\hline $\operatorname{beta}_{(m+1)}$ & [people/min] & $\begin{array}{l}\text { Transition rate of renting one bike from Node } i \text { to one of } \\
\text { the other nodes except Node } i(m=0,1, \cdots, K-1)\end{array}$ \\
\hline$\alpha$ & [people/min] & A certain value of the transition rate \\
\hline$\beta$ & [people/min] & A certain value of the transition rate \\
\hline$p_{l}$ & - & $\begin{array}{l}\text { Probability of having } l \text { bikes at Node } i \text { with no relocation } \\
(l=0,1, \cdots, K)\end{array}$ \\
\hline$\alpha_{i\left(m_{1}, m_{2}\right)}$ & [people/min] & $\begin{array}{l}\text { Transition rate of renting one bike at the virtual node and } \\
\text { returning it at node } i\left(m_{1}, m_{2}=0,1, \cdots, K-1\right)\end{array}$ \\
\hline$\alpha_{j\left(m_{1}, m_{2}\right)}$ & [people/min] & $\begin{array}{l}\text { Transition rate of renting one bike at the virtual node and } \\
\text { reurning it at node } j\left(m_{1}, m_{2}=0,1, \cdots, K-1\right)\end{array}$ \\
\hline
\end{tabular}




\begin{tabular}{|c|c|c|}
\hline Notation & Units & Definitions \\
\hline$\beta_{i\left(m_{1}, m_{2}\right)}$ & [people/min] & $\begin{array}{l}\text { Transition rate of renting one bike at Node and returning it } \\
\text { at the virtual node } i\left(m_{1}, m_{2}=0,1, \cdots, K-1\right)\end{array}$ \\
\hline$\beta_{j\left(m_{1}, m_{2}\right)}$ & [people/min] & $\begin{array}{l}\text { Transition rate of renting one bike at Node and returning it } \\
\text { at the virtual node } j\left(m_{1}, m_{2}=0,1, \cdots, K-1\right)\end{array}$ \\
\hline$\alpha_{i} \beta_{j\left(m_{1}, m_{2}\right)}$ & [people/min] & $\begin{array}{l}\text { Transition rate of renting one bike at Node and returning it } \\
\text { at node } i\left(m_{1}, m_{2}=0,1, \cdots, K-1\right)\end{array}$ \\
\hline$\alpha_{j} \beta_{i\left(m_{1}, m_{2}\right)}$ & [people/min] & $\begin{array}{l}\text { Transition rate of renting one bike at Node and returning it } \\
\text { at node } j\left(m_{1}, m_{2}=0,1, \cdots, K-1\right)\end{array}$ \\
\hline$p_{m_{1}}$ & - & Probability of having $m_{1}$ bikes at node $i\left(m_{1}=0,1, \cdots, K\right)$ \\
\hline$p_{m_{2}}$ & - & Probability of having $m_{2}$ bikes at node $j\left(m_{2}=0,1, \cdots, K\right)$ \\
\hline$p_{m_{1}, m_{2}}$ & - & $\begin{array}{l}\text { Probability of having } m_{1} \text { bikes at node } i \text { and having } m_{2} \\
\text { bikes at node } j \text { with no relocation }\left(m_{1}, m_{2}=0,1, \cdots, K\right)\end{array}$ \\
\hline$p_{l}^{\prime}$ & - & $\begin{array}{l}\text { Adjusted probability of having } l \text { bikes at node } i \text { with } \\
\text { relocation }(l=0,1, \cdots, K)\end{array}$ \\
\hline$r$ & [times] & Relocation frequency during operation time \\
\hline$c$ & - & $\begin{array}{l}\text { Coefficient of variation in probability of having zero bikes } \\
\text { at node } i \text { under the influence of relocation }\end{array}$ \\
\hline$\mu$ & - & $\begin{array}{l}\text { Index of measuring the influence of relocation on } \\
\text { steady-state probabilities }\end{array}$ \\
\hline$B$ & - & $\begin{array}{l}\text { Coefficient of variation in probability of having bikes at } \\
\text { node } i \text { under the influence of relocation }\end{array}$ \\
\hline$c_{\text {in }}$ & $\begin{array}{l}{[\mathrm{RMB}} \\
\cdot \mathrm{min} /(\text { bike} \cdot \text { person) }]\end{array}$ & Unit revenue per bike per person during operation time \\
\hline$c_{\mathrm{re}}$ & [RMB / time $]$ & Unit cost of one-time relocation \\
\hline$c_{p}$ & {$[\mathrm{RMB} \cdot \mathrm{min} /$ person] } & $\begin{array}{l}\text { Penalty per person for unmet demands during operation } \\
\text { time }\end{array}$ \\
\hline$Y(r)$ & {$[\mathrm{RMB}]$} & Total profit with relocation \\
\hline$r_{\max }$ & [times] & $\begin{array}{l}\text { Optimal relocation frequency which corresponds to the } \\
\text { maximum total profit with relocation }\end{array}$ \\
\hline$Y\left(r_{\max }\right)$ & {$[\mathrm{RMB}]$} & $\begin{array}{l}\text { Maximum total profit with relocation by adopting the } \\
\text { optimal relocation frequency }\end{array}$ \\
\hline
\end{tabular}

\subsection{A Markovian Queueing Network with Higher Demands}

Samet et al. study the possibility of applying a model reduction method to a closed queueing network [24]. The aim is to reduce the complexity of the network model. Because when the network model is used to model a dockless bike-sharing system, both the excessive nodes and their intricate relationship increase the burden of calculation rapidly and make the problem more difficult to solve. Considering the potential barrier, a model reduction method is adopted based on the basic idea proposed by Samet et al. in this work [24]. The main idea of this method is to aggregate multiple nodes into a virtual single node. The additive property of independent Poisson random variables demonstrates its reliability from a demand-side perspective. If taking a 3-node network as an example, we reduce a 3-node system into a 2-node system with a virtual node (i.e., a combination of two of the nodes). It is shown by the results that the aggregation of two nodes reduces the state space of the network significantly, from a three-tuple state space: $(K, 0,0), \ldots,(0, K, 0), \ldots,(0,0, K)$ to a two-tuple space: $(K, 0),(K-1,1), \ldots,(1, K-1),(0, K)$. More generally, a simplified model with one single node (i.e., node $i$ ) and a combination of $N-1$ nodes as a virtual node is shown in Figure (1). 


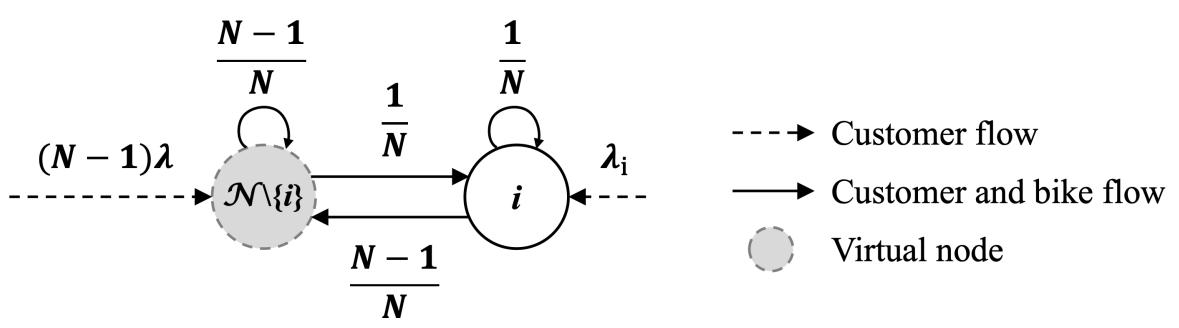

Figure 1. A reduced $N$-node network with the same probabilities and the same arrival rates at the nodes which are aggregated into a virtual node.

In this subsection, we build network models based on the model reduction method and differentiate a node with higher demands from the other nodes (i.e., $\lambda \leq \lambda_{i}$ ). We assume that it is possible to have zero bikes at the nodes which are inside a virtual node during the state transition process of the dockless bike-sharing system (see Figure 2). The digits in circles represent the changing number of bikes at the high-demand node. The number of bikes at a virtual node is $K$ minus the circled number. Therefore, each circled number can be described as a state of the system. $\alpha_{(0)}, \alpha_{(1)}, \cdots, \alpha_{(K-1)}$ and $\beta_{(1)}, \beta_{(2)}, \cdots, \beta_{(K)}$ are the state transition rates. $\left\{\alpha_{(m)}, m=0,1, \cdots, K-1\right\}$ means the rate of returning one bike, which is picked up at one of the nodes from the virtual node, at Node $i$. $\left\{\beta_{(m+1)}, m=0,1, \cdots, K-1\right\}$ means renting one bike at Node $i$ and returning it at one of the other nodes except node $i$. Although the complexity increases with the increasing number of nodes in the system, general formulas are concluded:

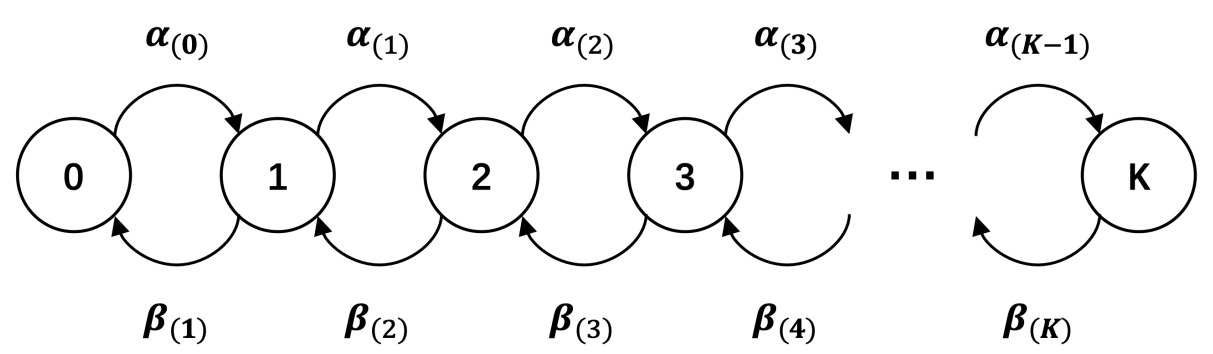

Figure 2. The state transition diagram with a high-demand node.

A 3-node system:

$$
\alpha_{(m)}=\frac{2}{3} \lambda \frac{K-m}{K-m+1}, \quad m=0,1, \cdots, K
$$

A 4-node system:

$$
\alpha_{(m)}=\frac{3}{4} \lambda \frac{K-m}{K-m+2}, \quad m=0,1, \cdots, K
$$

A $N$-node system:

$$
\alpha_{(m)}=\frac{N-1}{N} \lambda \frac{K-m}{K-m+N-2}, \quad m=0,1, \cdots, K
$$

$\beta_{(m+1)}$ is given by

$$
\beta_{(m+1)}=\lambda_{i} \frac{N-1}{N}, m=0,1, \cdots, K-1
$$


The steady-state probabilities are given by

$$
p_{l}=\frac{\alpha_{(m)}}{\beta_{(m+1)}} p_{l-1},(l, m)=\{(1,0), \cdots,(K, K-1)\}
$$

Based on $\sum_{l=0}^{K} p_{l}=1$, the formula of $p_{0}$ is yielded by

$$
p_{0}=\frac{1}{1+\frac{\lambda}{\lambda_{i}} \frac{K}{K+N-2}+\frac{\lambda^{2}}{\lambda_{i}^{2}} \frac{K(K-1)}{(K+N-2)(K+N-3)}+\cdots+\frac{\lambda^{K}}{\lambda_{i}^{K}} \frac{K !}{(K+N-2) \cdots(N-1)}}
$$

which leads to the formula of $p_{l}(l=1,2, \cdots, K)$ :

$$
p_{l}=\frac{\left(\frac{\lambda}{\lambda_{i}}\right)^{l} \frac{K(K-1) \cdots(K-l+1)}{(K+N-2)(K+N-3) \cdots(K+N-l-1)}}{1+\frac{\lambda}{\lambda_{i}} \frac{K}{K+N-2}+\frac{\lambda^{2}}{\lambda_{i}^{2}} \frac{K(K-1)}{(K+N-2)(K+N-3)}+\cdots+\frac{\lambda^{K}}{\lambda_{i}^{K}} \frac{K !}{(K+N-2) \cdots(N-1)}}
$$

The value of $p_{0}$ tends to be 1 when $\lambda_{i}$ is far larger than $\lambda$.

\subsection{A Markovian Queueing Network with Higher Demands and Lower Demands}

In reality, a bike-sharing system may include multiple nodes with complex and diverse demands from customers. In order to make the models more related to a real-world network, the Markovian queueing network model is extended in the paper to analyze the system under the influence of unequal demands, which include the higher demands and the lower demands. The reduced $N$-node network of the extension model is shown in Figure (3). Node $i$ refers to a node with higher demands, and Node $j$ refers to a node with lower demands than the nodes (whose arrival rates are the same and represented by $\lambda$ ) inside a virtual node (i.e., $\lambda_{j} \leq \lambda \leq \lambda_{i}$ ).

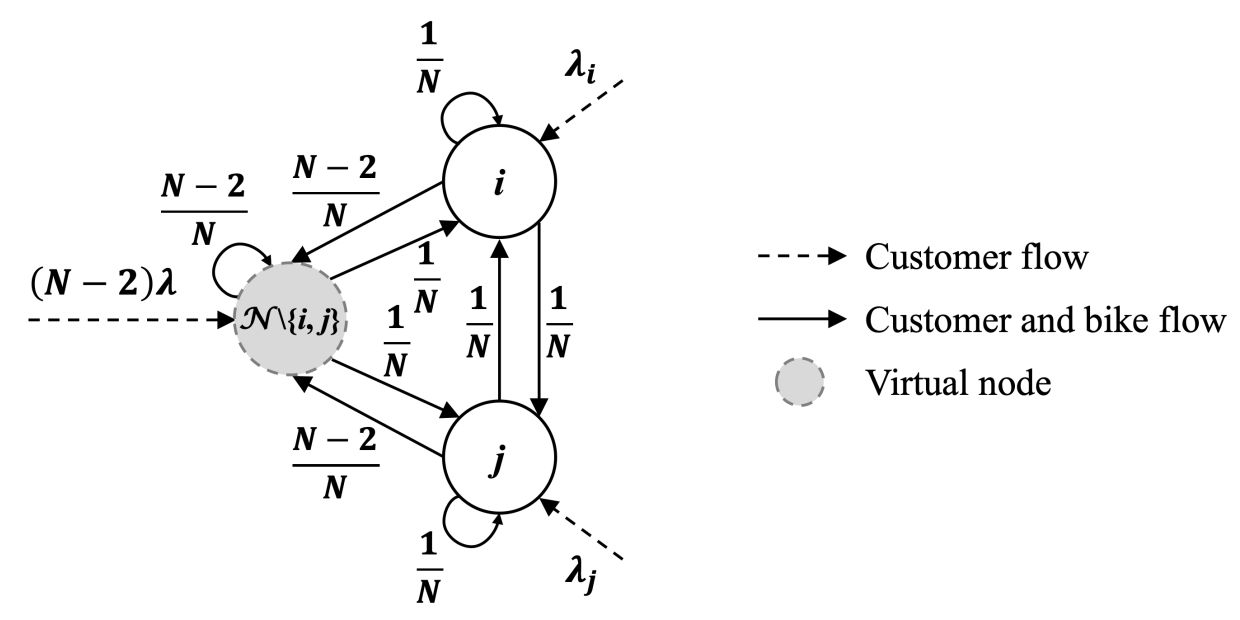

Figure 3. A reduced $N$-node network with the same probabilities and unequal demands.

With the existence of a virtual node and two single nodes, a three-tuple state space $\{(K, 0,0), \cdots,(0, K, 0), \cdots,(0,0, K)\}$ is used to represent the changing states of the whole system. Thanks to the fixed total number of bikes, a two-tuple state space $\{(K, 0),(K-1,1), \ldots,(1, K-$ $1),(0, K)\}$ performs a similar function, which represents the number of bikes at Node and Node separately (see Figure 4(a) and 4(b)). Each transition of the states means transferring one bike at a time. The six types of transition rates are defined as follows: 


$$
\begin{array}{ll}
\beta_{i\left(m_{1}+1, m_{2}\right)}=\beta_{i}=\lambda_{i} \frac{N-2}{N}, & \forall m_{1}, m_{2} \\
\beta_{j\left(m_{1}, m_{2}+1\right)}=\beta_{j}=\lambda_{j} \frac{N-2}{N}, & \forall m_{1}, m_{2} \\
\alpha_{j} \beta_{i\left(m_{1}+1, m_{2}\right)}=\frac{\lambda_{i}}{N}, & \forall m_{1}, m_{2} \\
\alpha_{i} \beta_{j\left(m_{1}, m_{2}+1\right)}=\frac{\lambda_{j}}{N}, & \forall m_{1}, m_{2} \\
\alpha_{i\left(m_{1}, m_{2}\right)}=\frac{N-2}{N} \frac{\lambda\left(K-m_{1}-m_{2}\right)}{K-m_{1}-m_{2}+N-3} & \\
\alpha_{j\left(m_{1}, m_{2}\right)}=\frac{N-2}{N} \frac{\lambda\left(K-m_{1}-m_{2}\right)}{K-m_{1}-m_{2}+N-3} &
\end{array}
$$

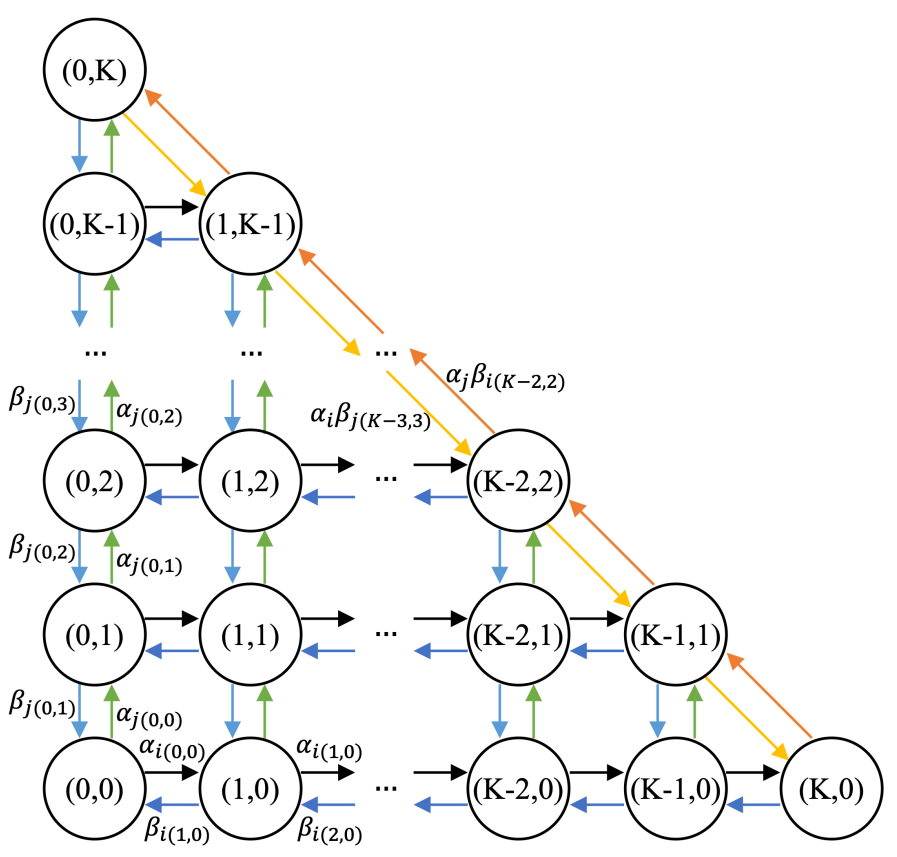

(a) The state transition diagram with unequal demands;

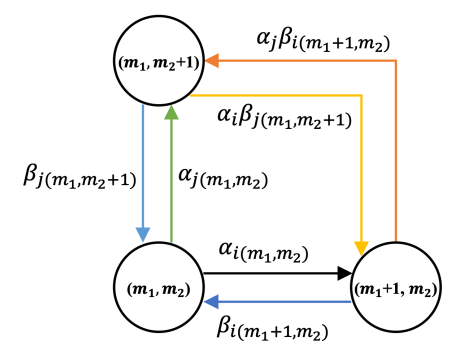

(b) The schematic symbols.

Figure 4. The detailed state transition diagram.

According to a main principle that the sum of the values of inflows equals to the sum of the values of outflows in a steady-state system, multiple equations (which are centered on each steady state and its directly-connected states) can be deducted. Equations are given as follows $\left(1 \leq m_{1}, m_{2} \leq K-1\right)$ :

$$
\begin{aligned}
& \left(\alpha_{i(0,0)}+\alpha_{j(0,0)}\right) p_{(0,0)}=\beta_{i} p_{1,0}+\beta_{j} p_{0,1} \\
& \left(\beta_{j}+\frac{\lambda_{j}}{N}\right) p_{0, K}=\frac{\lambda_{i}}{N} p_{1, K-1}+\alpha_{j(0, K-1)} p_{0, K-1} \\
& \left(\beta_{i}+\frac{\lambda_{i}}{N}\right) p_{K, 0}=\frac{\lambda_{j}}{N} p_{K-1,1}+\alpha_{i(K-1,0)} p_{K-1,0} \\
& \left(\beta_{i}+\beta_{j}+\alpha_{i\left(m_{1}, m_{2}\right)}+\alpha_{j\left(m_{1}, m_{2}\right)}\right) p_{m_{1}, m_{2}}
\end{aligned}
$$




$$
\begin{gathered}
=\beta_{i} p_{m_{1}+1, m_{2}}+\beta_{j} p_{m_{1}, m_{2}+1}+\alpha_{i\left(m_{1}-1, m_{2}\right)} p_{m_{1}-1, m_{2}}+\alpha_{j\left(m_{1}, m_{2}-1\right)} p_{m_{1}, m_{2}-1}, \quad \forall m_{1}+m_{2} \leq K-1 \\
\left(\beta_{j}+\alpha_{i\left(0, m_{2}\right)}+\alpha_{j\left(0, m_{2}\right)}\right) p_{0, m_{2}}=\beta_{i} p_{1, m_{2}}+\beta_{j} p_{0, m_{2}+1}+\alpha_{j\left(0, m_{2}-1\right)} p_{0, m_{2}-1} \\
\left(\beta_{i}+\alpha_{i\left(m_{1}, 0\right)}+\alpha_{j\left(m_{1}, 0\right)}\right) p_{m_{1}, 0}=\beta_{j} p_{m_{1}, 1}+\beta_{i} p_{m_{1}+1,0}+\alpha_{i\left(m_{1}-1,0\right)} p_{m_{1}-1,0} \\
\left(\beta_{i}+\frac{\lambda_{i}}{N}+\beta_{j}+\frac{\lambda_{j}}{N}\right) p_{m_{1}, K-m_{1}} \\
\quad=\frac{\lambda_{i}}{N} p_{m_{1}+1, K-m_{1}-1}+\frac{\lambda_{j}}{N} p_{m_{1}-1, K-m_{1}+1}+\alpha_{i\left(m_{1}-1, K-m_{1}\right)} p_{m_{1}-1, K-m_{1}}+\alpha_{j\left(m_{1}, K-m_{1}-1\right)} p_{m_{1}, K-m_{1}-1}
\end{gathered}
$$

In addition, there is a constraint toward the sum of the steady-state probabilities:

$$
\sum_{\substack{m_{1}, m_{2} \geq 0 \\ m_{1}+m_{2} \leq K}} p_{m_{1}, m_{2}}=1
$$

A unique solution of the steady-state probabilities can be obtained by solving the set of equations. The probabilities of having $m_{1}$ bikes at Node $i$ and the probabilities of having $m_{2}$ bikes at Node $j$ are given by the following formulas:

$$
\begin{aligned}
& p_{m_{1}}=\sum_{m_{2}=0}^{K-m_{1}} p_{m_{1}, m_{2}} \\
& p_{m_{2}}=\sum_{m_{1}=0}^{K-m_{2}} p_{m_{1}, m_{2}}
\end{aligned}
$$

Since it is a problem of solving non-homogeneous linear equations, we can use matrix inversion or other efficient algorithms to compute the solutions. In this paper, we use the open source $\mathrm{R}$ statistics software to obtain the values of steady-state probabilities. The limitation is that the burden of computation becomes huger with the increase of $K$.

\section{Optimization Methods}

According to the probabilistic results of the Markovian network model with one high-demand node, shared bikes are likely to leave the high-demand node and gather at the relatively low-demand nodes in the system in the long run. The basic relocation strategy we tend to adopt is to relocate the extra bikes from the relatively low-demand nodes (which have been aggregated into a virtual node in the network model with one high-demand node) to the high-demand node (which is represented as Node $i$ in the network model with one high-demand node) to reach the initial number of bikes at each node. The relocation strategy can be implemented as many times as needed during normal operation of the bike-sharing system and the relocation frequency can reflect the number of times of relocation.

In this paper, we assume that a more effective relocation strategy is able to reduce the value of $p 0$ more rapidly, which can be described as $p_{0}^{\prime} \propto c r^{-\mu} p_{0}(c>0, \mu>0) \cdot p_{0}^{\prime}$ represents the steady-state probability of having zero bikes at Node $i$ under the influence of relocation. The formulas and descriptions are based on the Markovian queueing network model with higher demands. When operators implement the relocation strategy more frequently, $p_{0}^{\prime}$ decreases from a basic value of $p_{0}$. To determine the relationship between the probabilities without relocation and the probabilities with relocation, we have $p_{0}^{\prime}=c r^{-\mu} p_{0}$. When $p_{0}^{\prime}$ equals to $p_{0}, c r^{-\mu}=1$, where the value of $r$ represents no relocation is implemented in the system. $r>c^{\frac{1}{\mu}}$ means the operators start to relocate bikes. 
Considering that the sum of all the probabilities equals to 1 , the other probabilities are assumed to increase proportionately to satisfy the constraint, which are given by

$$
p_{l}^{\prime}=B p_{l}, \quad \forall l=1, \cdots, K
$$

For $\sum_{l=0}^{K} p_{l}^{\prime}=1$, we have

$$
\begin{aligned}
& B=\frac{1-c r^{-\mu} p_{0}}{1-p_{0}} \\
& p_{l}^{\prime}= \begin{cases}c r^{-\mu} p_{0}, & l=0 \\
\frac{1-c r^{-\mu} p_{0}}{1-p_{0}} p_{l}, & l=1, \cdots, K\end{cases}
\end{aligned}
$$

Our aim is to maximize the total profit obtained by the operators of the dockless bike-sharing system after implementing the relocation strategy. The operating revenue comes from the customers who rent the shared bikes and return them successfully without any trouble. In reality, the customers often have to pay for the time they spend using the bikes. We assume that the operating revenue is in proportion to the customer demand, the number of available bikes at each node and the unit price charged by the operators. If there is a high demand for shared bikes or there is a large number of available bikes in the area, it is likely to squeeze more revenue from the customers. Based on the adjusted probabilities of having a number of bikes at Node $i$, the operating revenue is given by

$$
\left[\sum_{l=0}^{K} p_{l}^{\prime} l \lambda_{i}+\sum_{l=0}^{K} p_{l}^{\prime}(K-l) \lambda\right] c_{i n}=\left[K \lambda+\sum_{l=0}^{K} p_{l}^{\prime} l\left(\lambda_{i}-\lambda\right)\right] c_{i n}
$$

The operating cost can be divided into the cost of relocations (which is assumed to be in proportion to the relocation frequency and the unit cost of implementing the relocation strategy) and the penalty derived from the unmet demands (which is assumed to be in proportion to the arrival rate, the possibility of having zero bikes at each node and the unit cost of penalties). Due to the given constraint for $r$, if no relocation is implemented in the system, the cost of relocations will not occur. According to the initial assumption that the nodes inside a virtual node are the same, it is assumed that when the total number of bikes at these nodes (i.e., the nodes which are aggregated into one virtual node) is less than the number of the nodes inside the virtual node, there may be no bike available at some of these nodes. If the arrival rate at the node where there is no bike available for customers to use is large or the possibility of having zero bikes at the node is high, the cost of penalty from unmet demands will increase. The sum of the operating cost is given by

$$
\left(r-c^{\frac{1}{\mu}}\right) c_{r e}+\left(p_{0}^{\prime} \lambda_{i}+\sum_{l=1}^{N-1} p_{K-N+1+l}^{\prime} l \lambda\right) c_{p}
$$

The total profit after implementing the relocation strategy can be calculated by the following formula:

$$
\begin{aligned}
\max Y\left(r_{\max }\right)=[K \lambda+ & \left.\sum_{l=0}^{K} p_{l}^{\prime}\left(r_{\max }\right) l\left(\lambda_{i}-\lambda\right)\right] c_{i n} \\
& \quad-\left(r_{\max }-c^{\frac{1}{\mu}}\right) c_{r e}-\left[p_{0}^{\prime}\left(r_{\max }\right) \lambda_{i}+\sum_{l=1}^{N-1} p_{K-N+1+l}^{\prime}\left(r_{\max }\right) l \lambda\right] c_{p}
\end{aligned}
$$

$Y(r)$ means the total profit with the corresponding relocation frequency $r . r_{\max }$ represents the optimal relocation frequency which corresponds to the maximum profit $Y\left(r_{\max }\right)$ that the operators can obtain after relocating the bikes in the system. It is found that the relationship between $Y(r)$ and $r$ takes the shape of a concave curve. Before the relocation frequency reaches the turning point, $Y(r)$ 
increases with the increase of $r$. After the turning point, decreases with the increase of . The turning point turns out to be the best choice for the relocation frequency and the total profit. Therefore, we take a derivative of the total profit with respect to the relocation frequency in order to get the formula of $r_{\max }$. The first-order partial derivative of Equation (29) with respect to $r$ is given by

$$
\frac{\partial Y(r)}{\partial r}=\sum_{l=1}^{K}\left(\lambda_{i}-\lambda\right) c_{i n} \frac{c \mu p_{0} l p_{l}}{1-p_{0}} r^{-\mu-1}+\lambda_{i} c_{p} c \mu p_{0} r^{-\mu-1}-\sum_{l=1}^{N-1} \lambda c_{p} \frac{c \mu p_{0} l p_{K-N+1+l}}{1-p_{0}} r^{-\mu-1}-c_{r e}
$$

When the first-order partial derivative equals to $0, r_{\max }$ can be calculated by the following formula

$$
r_{\max }=\left[\frac{\sum_{l=1}^{K}\left(\lambda_{i}-\lambda\right) c_{i n} \frac{c \mu p_{0} l p_{l}}{1-p_{0}}+\lambda_{i} c_{p} c \mu p_{0}-\sum_{l=1}^{N-1} \lambda c_{p} \frac{c \mu p_{0} l p_{K-N+1+l}}{1-p_{0}}}{c_{r e}}\right]^{\frac{1}{\mu+1}}
$$

\section{Results}

\subsection{Probabilistic Results}

The relationship between the steady-state probabilities and the arrival rates at different nodes in the network model with unequal demands is investigated in multiple cases by numerical experiments. The setting of the arrival rates and the corresponding figures of the cases are shown in Table 4-1.

Table 2. Setting of the arrival rates in the network model with unequal demands.

\begin{tabular}{lllll}
\hline Case ID & $\lambda$ & $\lambda_{i}$ & $\lambda_{j}$ & Figure \\
\hline Case 1 & 1 & 1 & 0.8 & Figure (5) \\
Case 2 & 1 & 1 & 0.6 & Figure (6) \\
Case 3 & 1 & 1 & 0.4 & Figure (7) \\
Case 4 & 1 & 1.2 & 0.8 & Figure (8) \\
Case 5 & 1 & 1.2 & 0.6 & Figure (9) \\
Case 6 & 1 & 1.2 & 0.4 & Figure (10) \\
Case 7 & 1 & 1.2 & 0.2 & Figure (11) \\
Case 8 & 1 & 1.2 & 0.1 & Figure (12) \\
Case 9 & 1 & 1.2 & 0.05 & Figure (13) \\
\hline
\end{tabular}




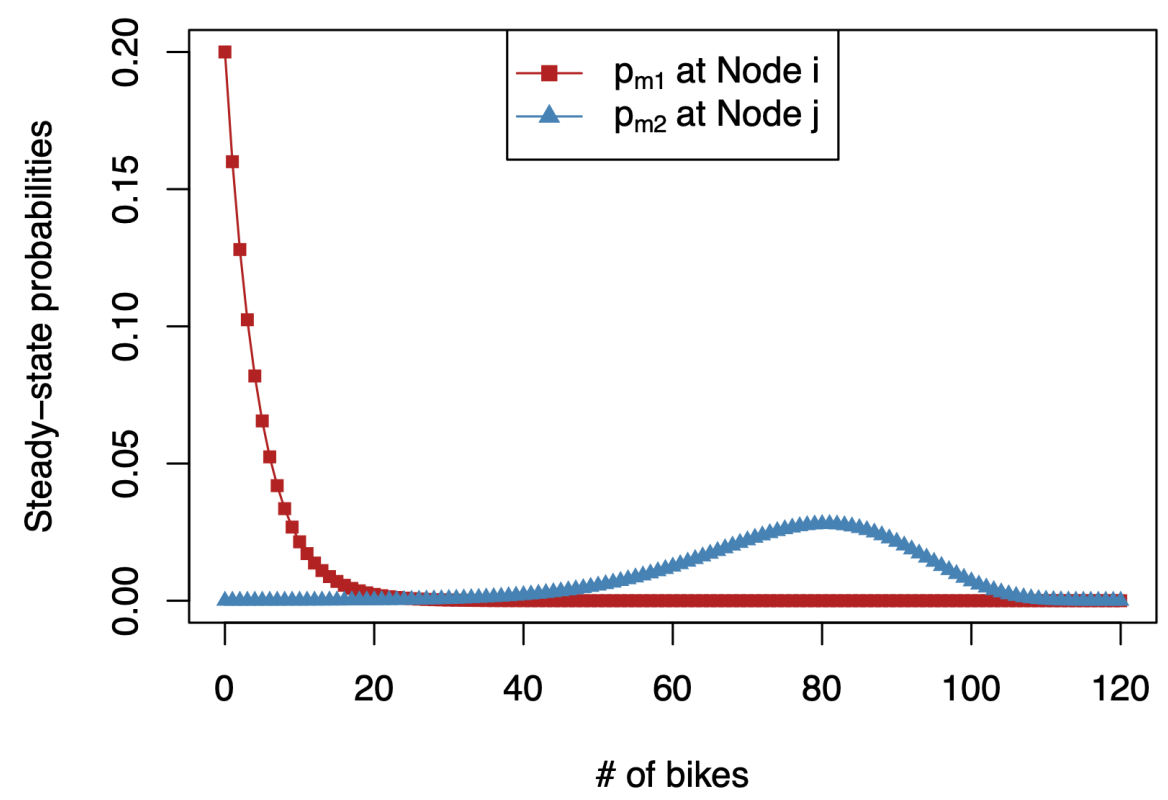

Figure 5. Probabilities with respect to the number of bikes at Node $i$ and $j$ separately in Case 1 .

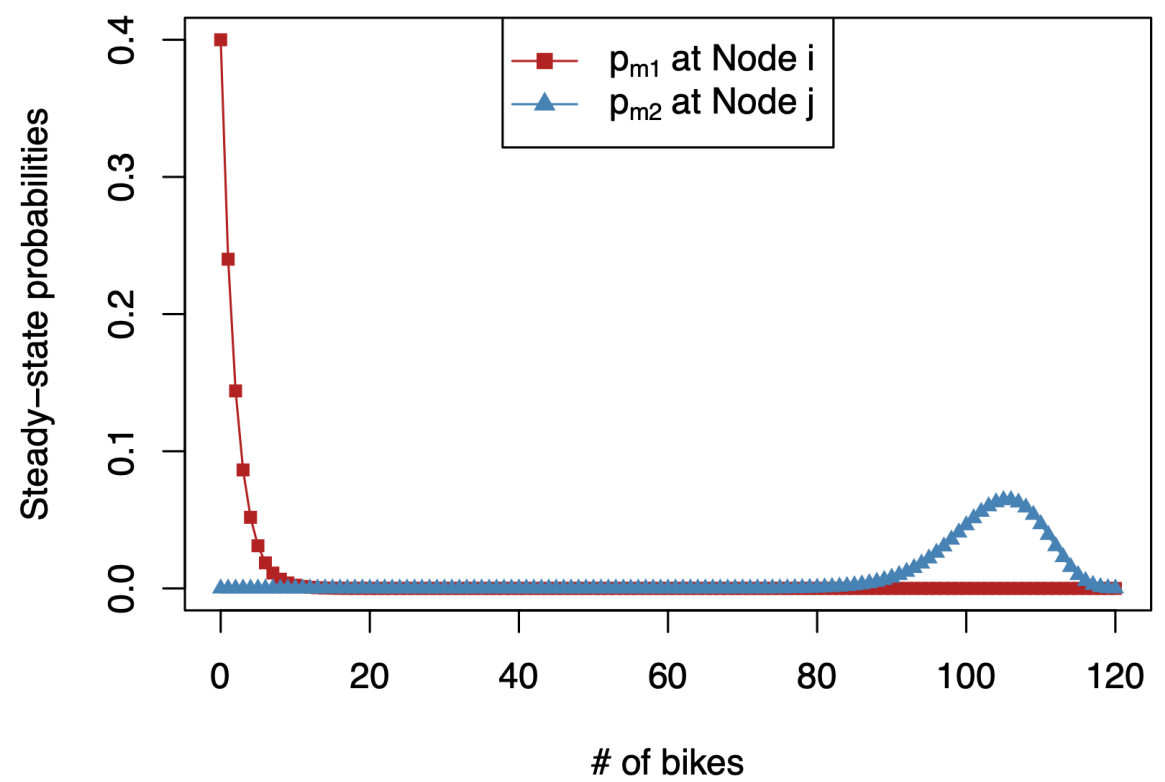

Figure 6. Probabilities with respect to the number of bikes at Node $i$ and $j$ separately in Case 2 . 


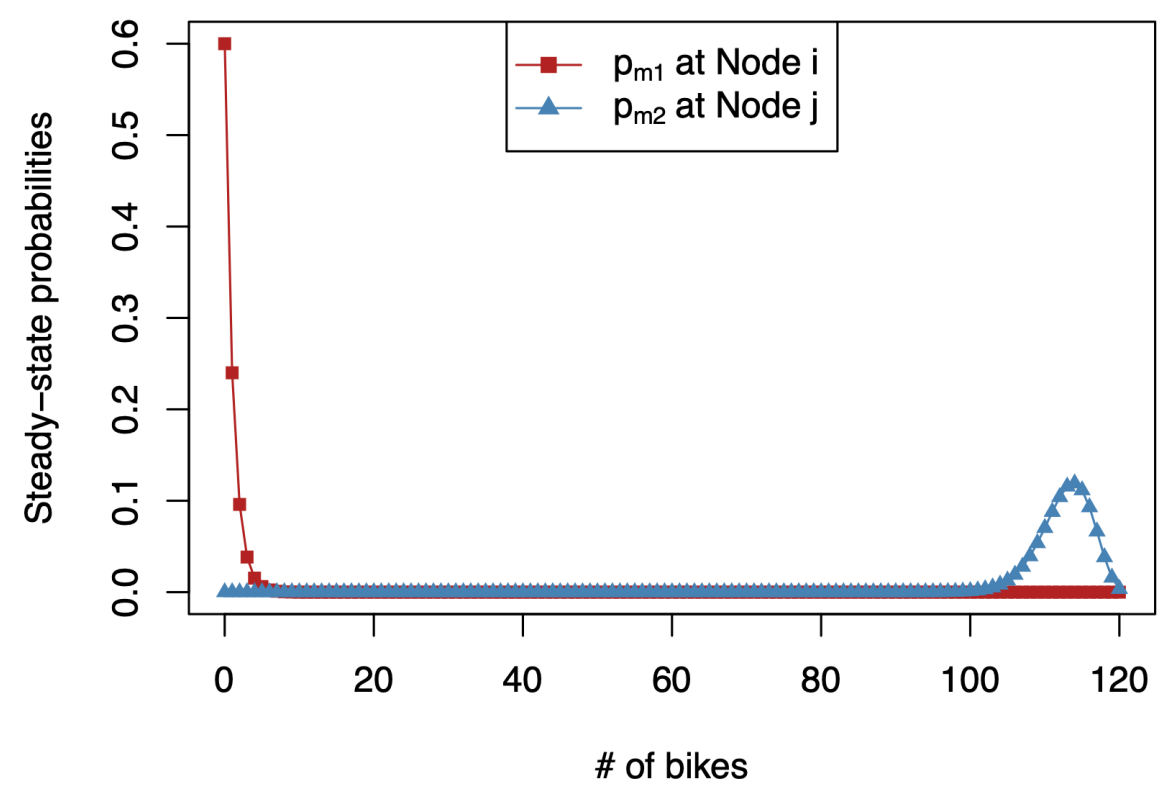

Figure 7. Probabilities with respect to the number of bikes at Node $i$ and $j$ separately in Case 3.

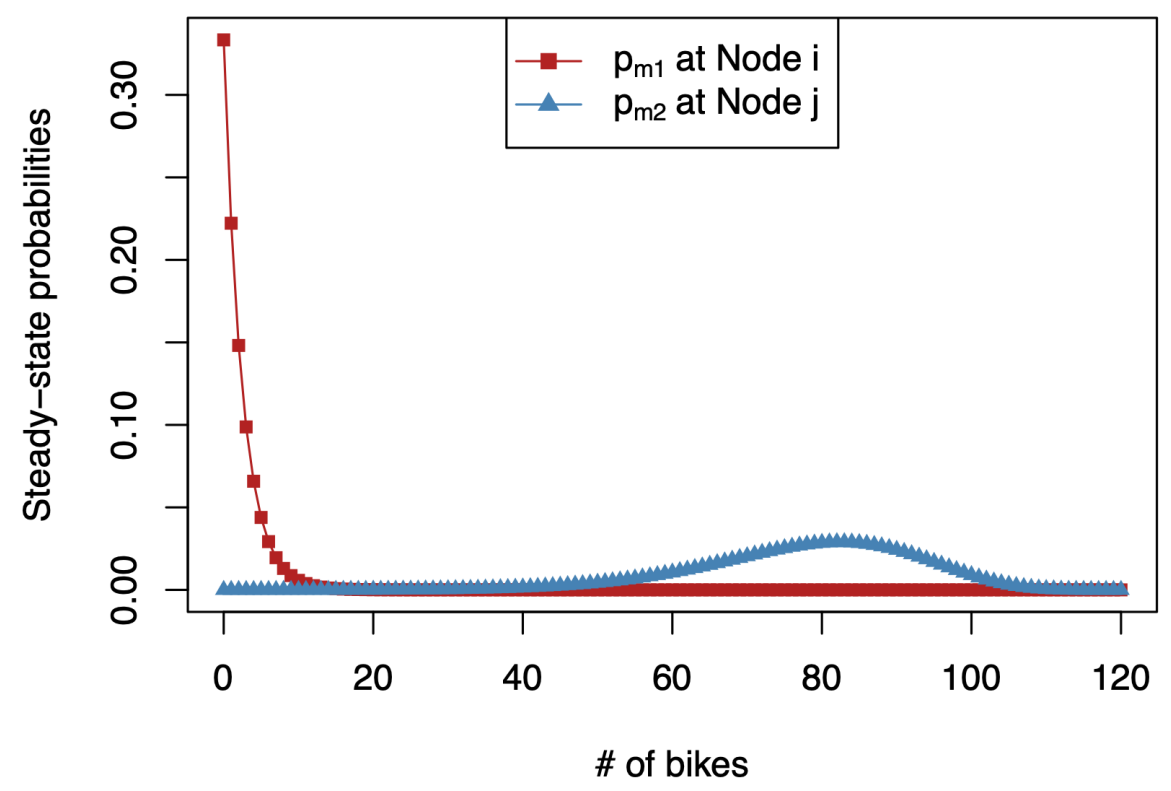

Figure 8. Probabilities with respect to the number of bikes at Node $i$ and $j$ separately in Case 4 . 


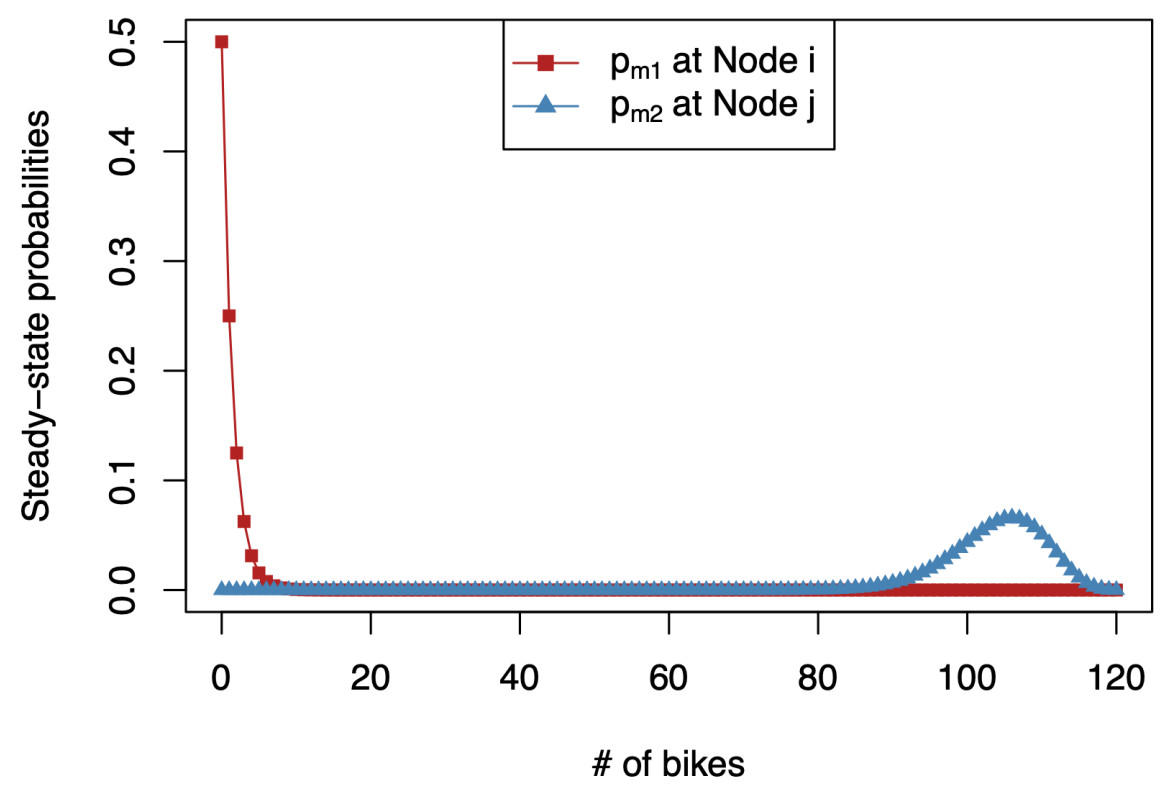

Figure 9. Probabilities with respect to the number of bikes at Node $i$ and $j$ separately in Case 5 .

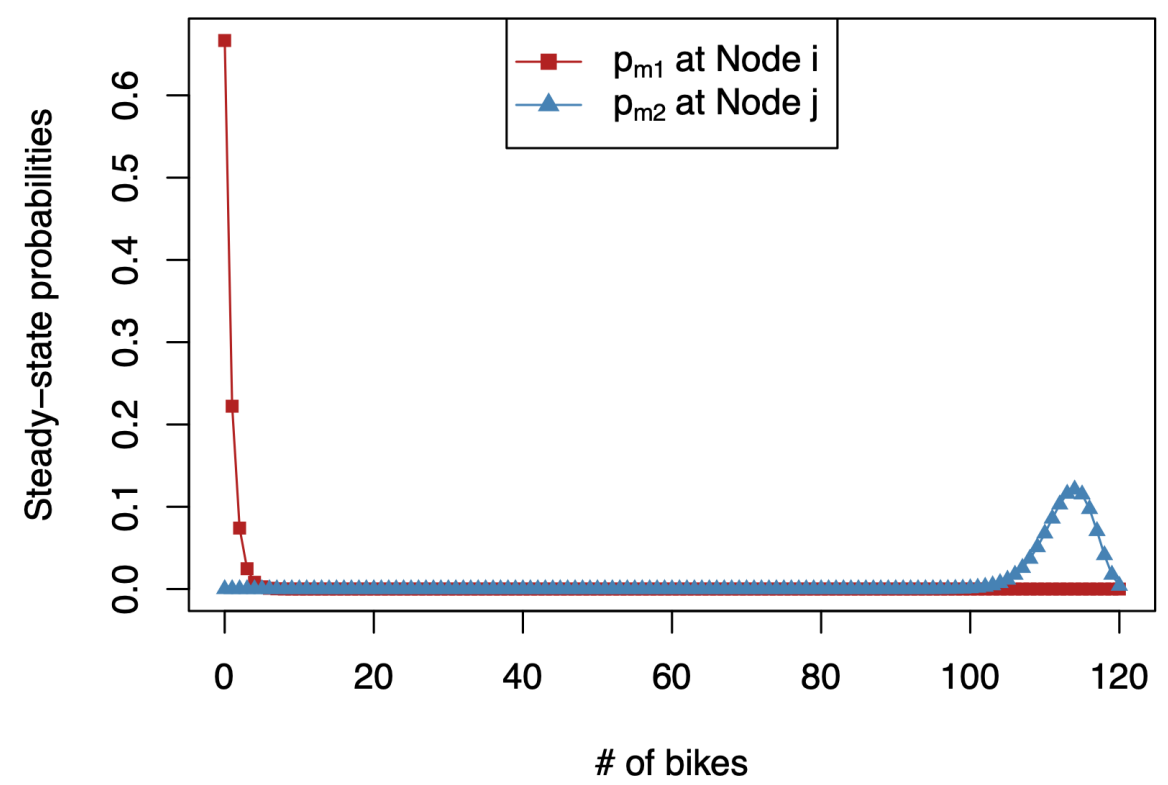

Figure 10. Probabilities with respect to the number of bikes at Node $i$ and $j$ separately in Case 6 . 


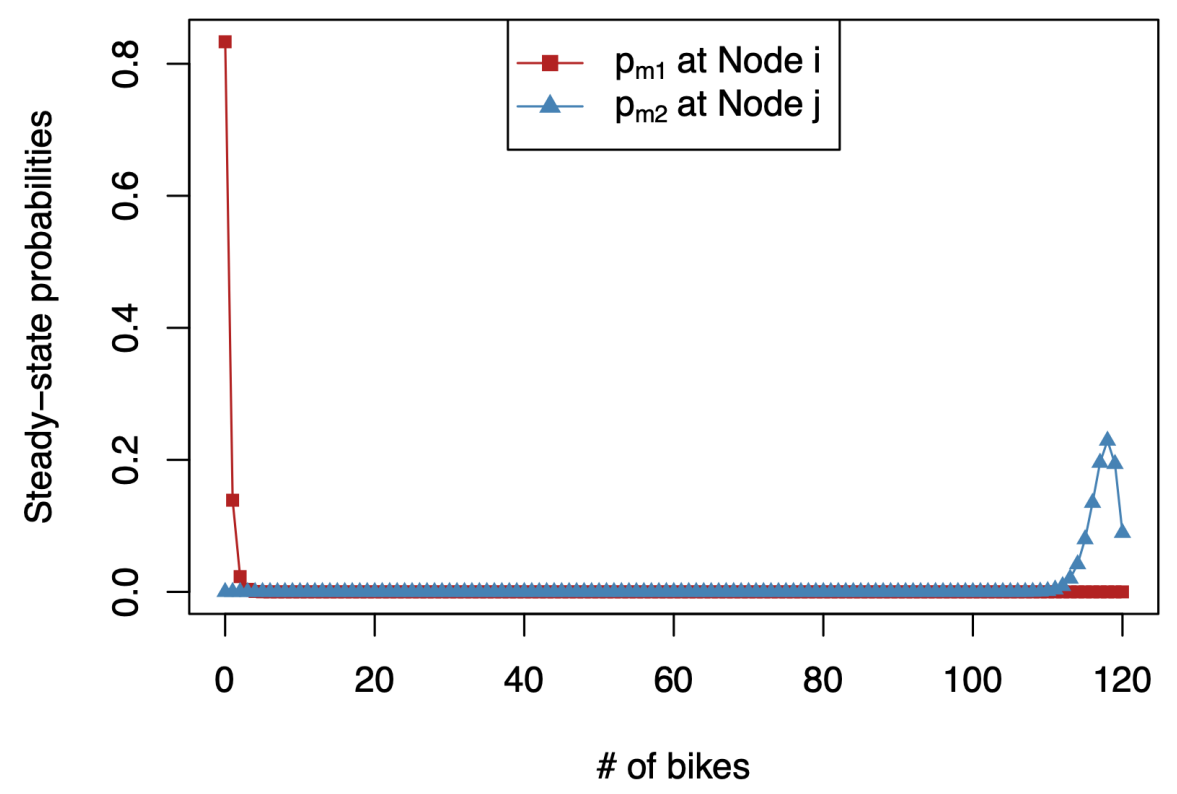

Figure 11. Probabilities with respect to the number of bikes at Node $i$ and $j$ separately in Case 7 .

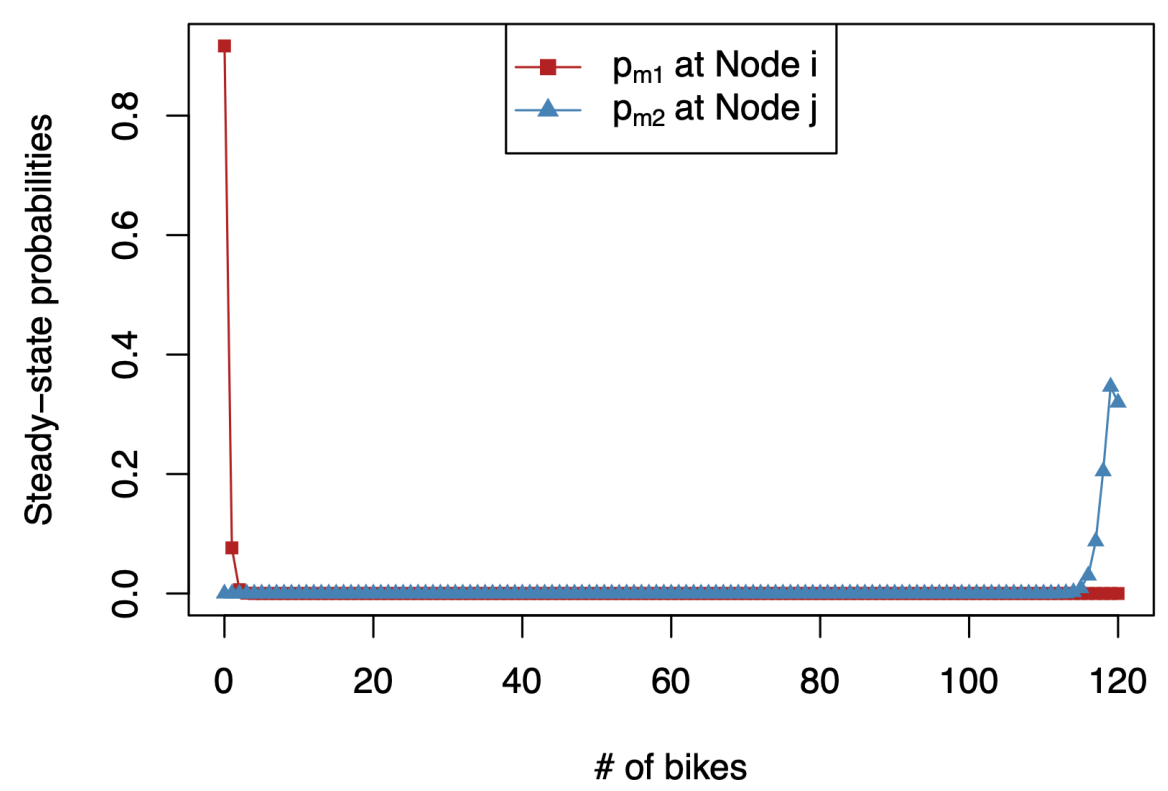

Figure 12. Probabilities with respect to the number of bikes at Node $i$ and $j$ separately in Case 8 . 


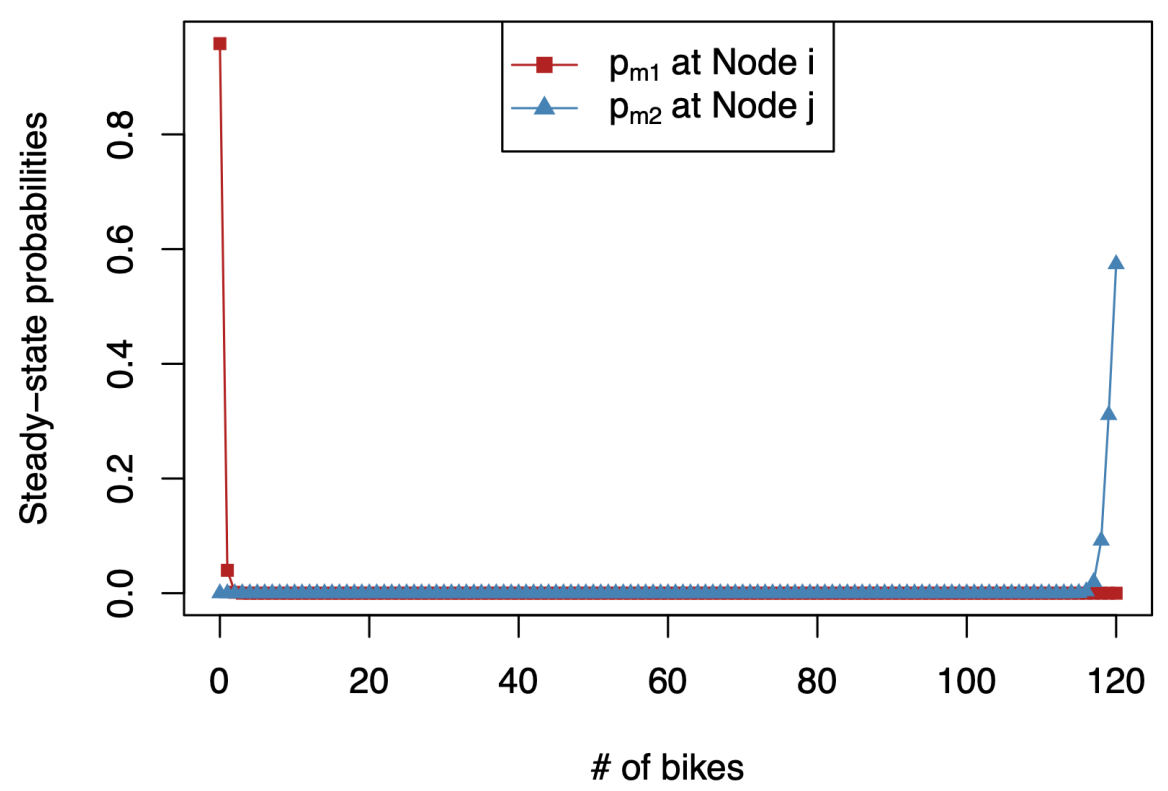

Figure 13. Probabilities with respect to the number of bikes at Node $i$ and $j$ separately in Case 9 .

It can be concluded from the change of the arrival rates at different nodes that the long-term bike distribution in the dockless bike-sharing system is more sensitive to the higher demands than the lower demands. Due to the difference, the number of bikes at the nodes with higher demands decreases more quickly and more likely than the increase of the number of bikes at the nodes with lower demands. For the nodes with middle demands, the number of bikes may decrease more mildly compared with the decreased number of bikes at the nodes with higher demands. It is suggested that the operators should pay more attention to the high-demand nodes, which may suffer the biggest loss in the system. Unless one low-demand node has the smallest arrival rate which is terribly different from the arrival rates at the other nodes, there is a relatively small possibility that all of the bikes in the system are transferred to one low-demand node in a limited period of time, which can be regarded as the biggest imbalance of bikes in the system.

\subsection{Profitability Results}

\subsubsection{The Effect of Relocation-related Parameters}

Both $c$ and $\mu$ are two positive parameters which can be regarded as a measure of the effectiveness of an adopted relocation strategy. If the relocation strategy is more effective, $c$ is supposed to decrease and $\mu$ is supposed to increase, which can contribute a low probability of having zero bikes at the high-demand node under the influence of relocations. In the numerical experiments, $c$ and $\mu$ are changed separately to analyze their influence on the performance of the optimization model, which is shown in Figure (14) and Figure (15). 


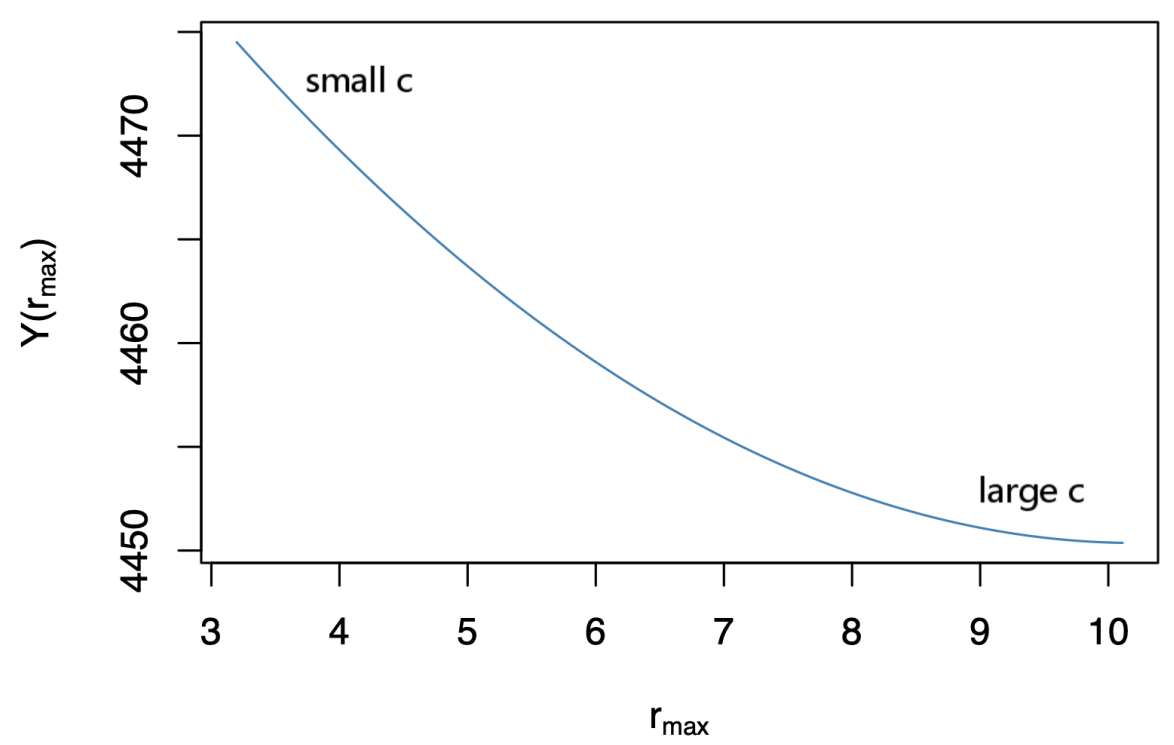

Figure 14. The maximum profit after implementing the relocation strategy with respect to the optimal relocation frequency under the influence of $c$.

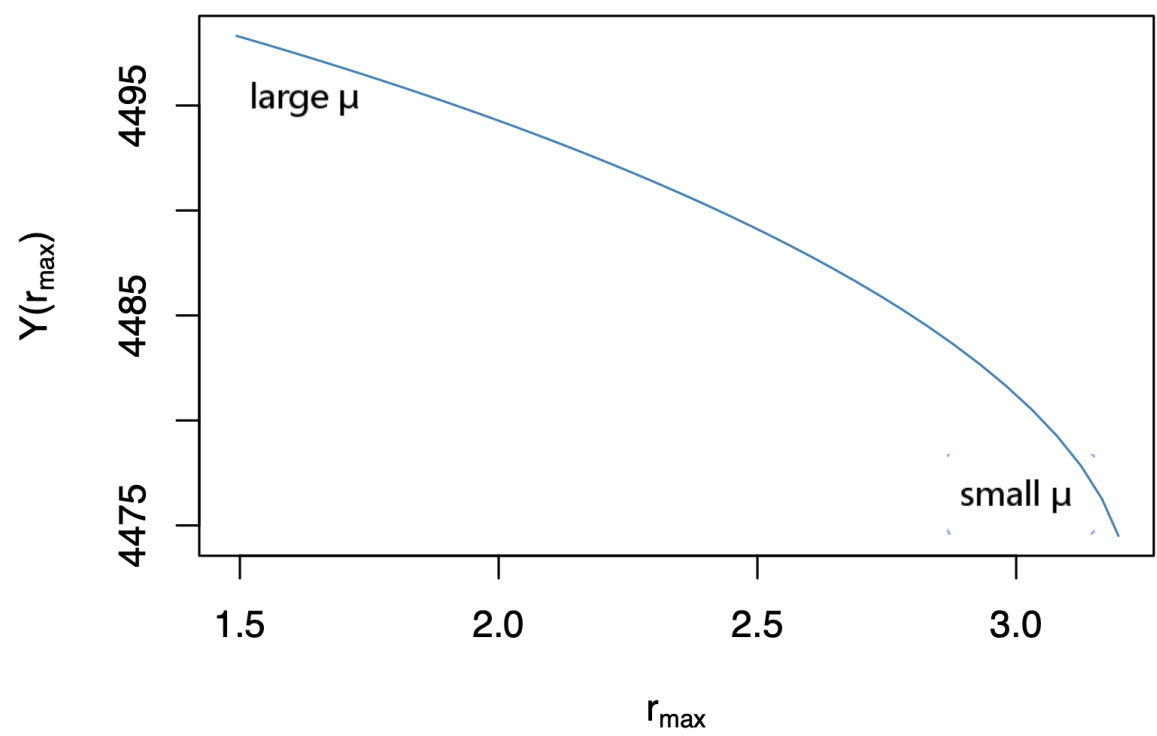

Figure 15. The maximum profit after implementing the relocation strategy with respect to the optimal relocation frequency under the influence of $\mu$.

The value of $Y\left(r_{\max }\right)$ decreases with the increase of $r_{\max }$ both in Figure (14) and Figure (15). When $c$ decreases or $\mu$ increases, the maximum profit tends to increase and the optimal relocation frequency tends to decrease. It is indicated that the relocation strategy does work well. An effective relocation strategy can reduce the relocation frequency and save the expense of frequent relocations, which can increase the total profit indirectly. However, the concavity and convexity of the curves shown in these two figures is different between $c$ and $\mu$. In Figure (14), $Y\left(r_{\max }\right)$ is more sensitive when the value of $c$ is small, and $r_{\max }$ is more sensitive when the value of $c$ is large. In Figure (15), $Y\left(r_{\max }\right)$ is more sensitive when the value of $\mu$ is small, and $r_{\max }$ is more sensitive when the value of $\mu$ is large. Since it is preferable for operators to gain more profits, a combination of a small $c$ and a large $\mu$ can properly decrease the sensitivity of $Y\left(r_{\max }\right)$ and $r_{\max }$ to the effectiveness of relocation strategies, which allows 
the operators to make more flexible decisions about what kind of relocation strategies they want to adopt.

\subsubsection{The Effect of Revenue-related and Cost-related Parameters}

$c_{i n}, c_{r e}$ and $c_{p}$ are parameters related to the operating revenue and the operating cost. As is shown in Figure (16) and Figure (17), $Y\left(r_{\max }\right)$ increases with the increase of $r_{\max }$ under the influence of $c_{i n}$ and $c_{r e} . Y\left(r_{\max }\right)$ increases with the decrease of $r_{\max }$ under the influence of $c_{p}$ in Figure (18). It can be explained by the different meanings of these parameters. The relocation strategy can reset the number of bikes at each node to an initial state, which make the bike-sharing system able to supply the customers with enough bikes after each relocation. Due to the definition of $c_{i n}$, the high price means operators can generate more revenue by satisfying the customer demand, which can be promised by frequent relocations. Therefore, with the increase of $c_{i n}$, both the optimal relocation frequency and the maximum profit increase. According to the change of $r_{\max }$ and $Y\left(r_{\max }\right)$ in Figure (16), $r_{\max }$ is insensitive to the increase of $c_{i n}$ and $Y\left(r_{\max }\right)$ is sensitive to the increase of $c_{i n}$. In reality, operators can make more profits by raising the unit price. But they have to consider the unit price given by their competitors, who have the ability to pull regular customers from them. Although acquiring a large revenue from customers can cover the expense on relocations to some extent, the increase of the optimal relocation frequency is slight. For operators, pricing decisions have a significant influence on the total profit but have a slight effect on the decision-making about relocating bikes. As is shown in Figure (17), with the increase of $c_{r e}$, both $r_{\max }$ and $Y\left(r_{\max }\right)$ decrease. It is because that the high cost of relocating bikes is supposed to decrease the relocation frequency to save cost for operators. With the decrease of relocation frequency, the total profit decreases due to the huge expense on relocations and the decreased revenue caused by the occasional shortage of bikes at some nodes. The slope of the curve in Figure (17) indicates that $r_{\max }$ is more sensitive to the change of $c_{r e}$ than $Y\left(r_{\max }\right)$, especially when $c_{r e}$ has a small value. It is suggested that operators should consider how to reduce the expense on relocating bikes in the system, which can be very beneficial to solve the imbalance problem of bike distribution. In Figure (18), $r_{\max }$ increases and $Y\left(r_{\max }\right)$ decreases with the increase of $c_{p}$. The large value of $c_{p}$ means a large penalty of losing customers, which happens when customers arrive at the node where there is no bike available. The increase of relocation frequency can minimize the losses by relocating bikes to the nodes with high demands. With the increase of the penalty and the increased cost derived from frequent relocations, the total profit decreases. In the real world, the unit cost of penalty from unmet demands is difficult to measure. In the numerical experiments, the value of $c_{p}$ is set far larger than the values of $c_{i n}$ and $c_{r e}$. It is because that unmet demands may have a significant impact on the loyalty of the regular customers. If the customers often cannot find any bikes to ride, they are likely to feel dissatisfied with the dockless bike-sharing system and turn for alternatives, which may bring about great losses (e.g., an obvious decrease in the potential revenue) for the bike-sharing company. Therefore, setting a large value of $c_{p}$ in the optimization model for a relocation problem can emphasize the need for a suitable relocation frequency. 


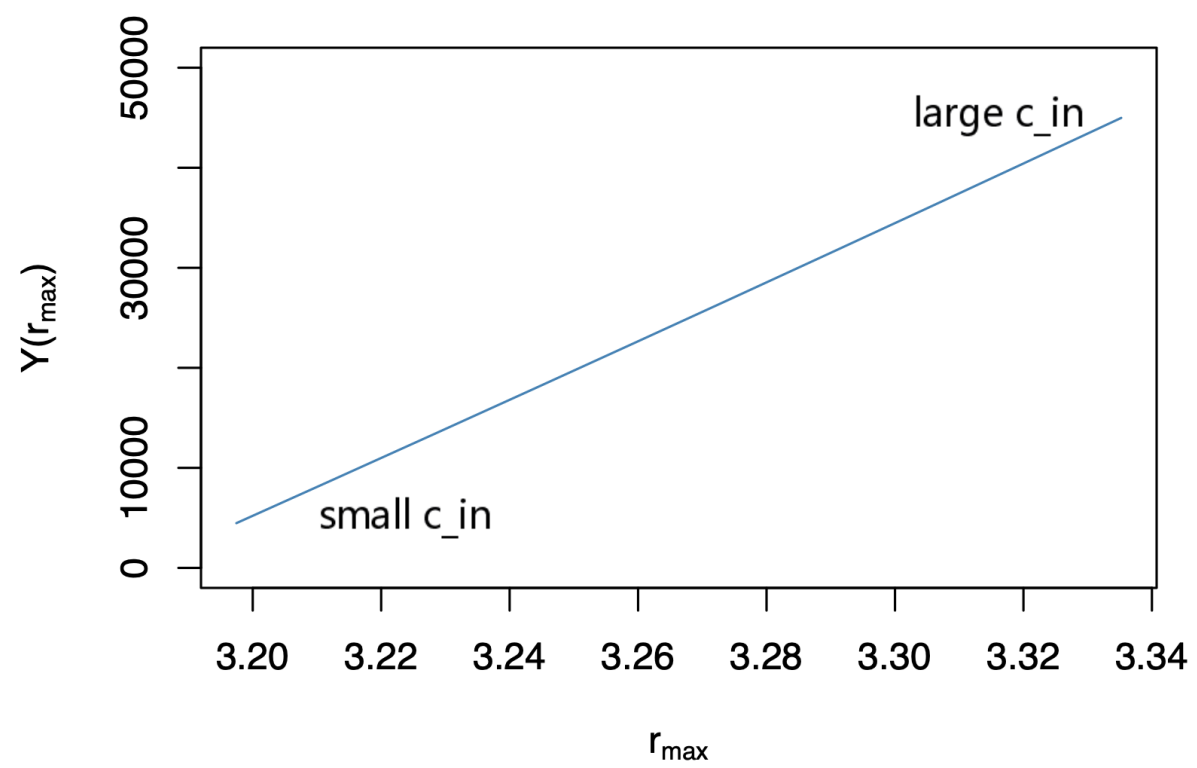

Figure 16. The maximum profit after implementing the relocation strategy with respect to the optimal relocation frequency under the influence of $c_{i n}$.

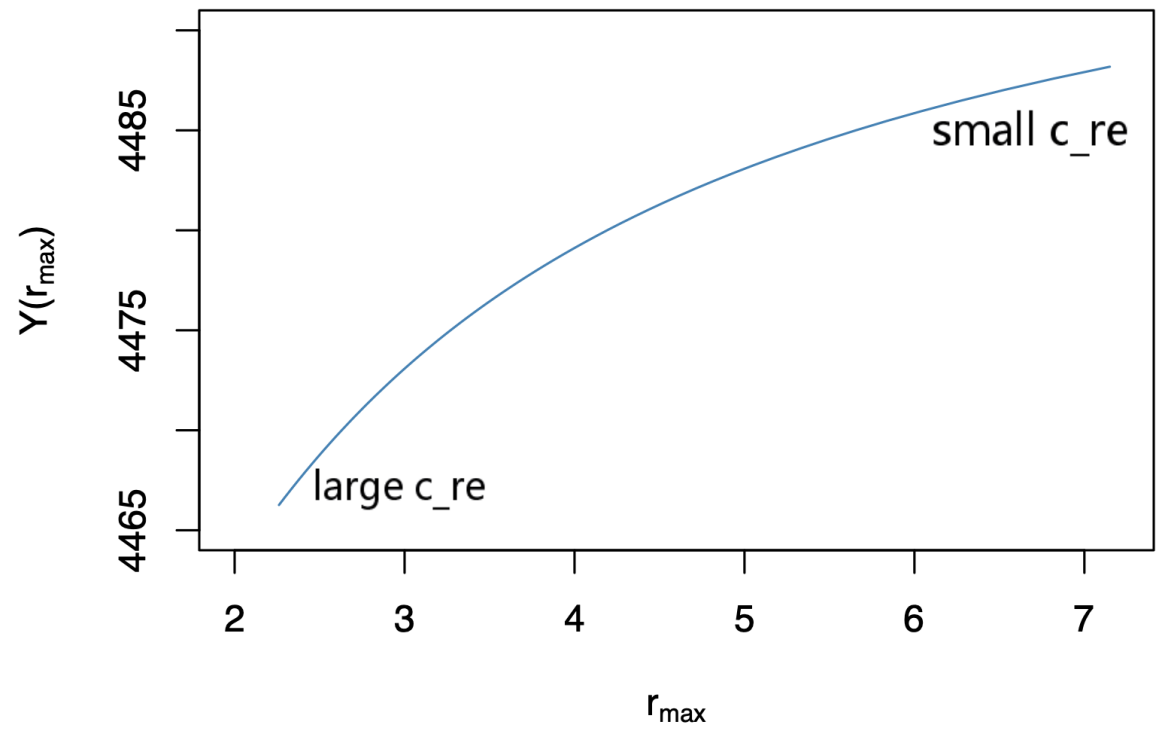

Figure 17. The maximum profit after implementing the relocation strategy with respect to the optimal relocation frequency under the influence of $c_{r e}$. 


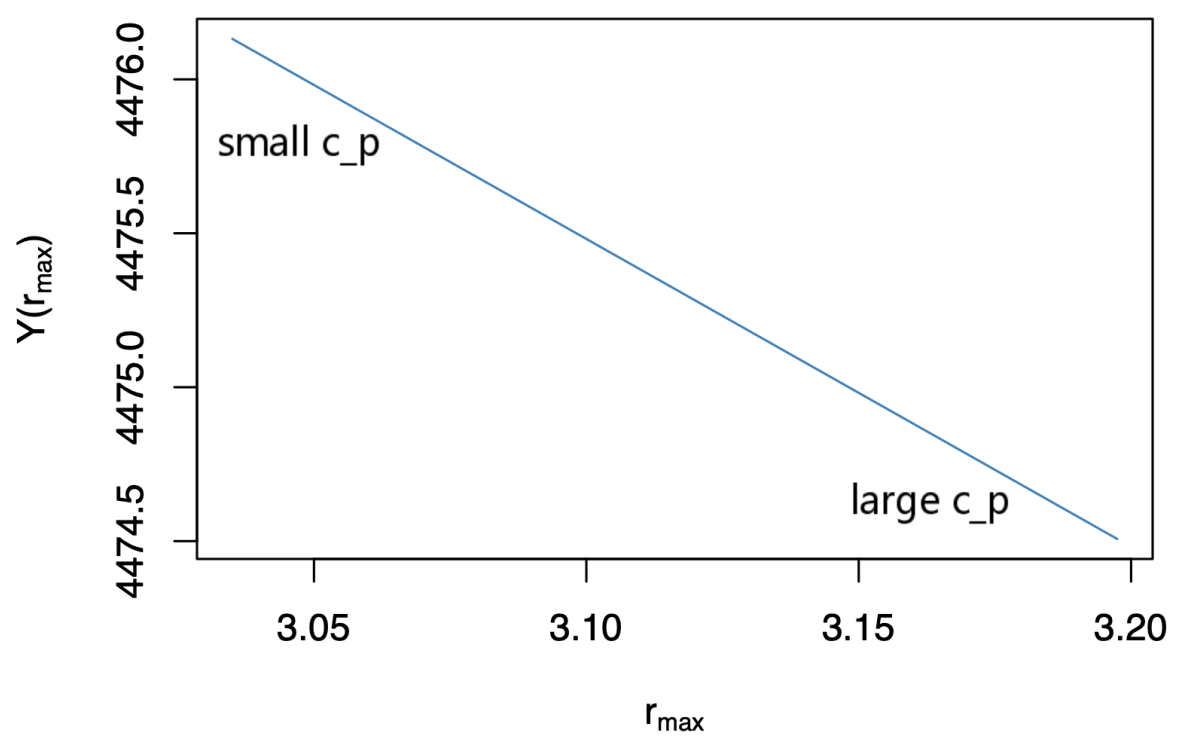

Figure 18. The maximum profit after implementing the relocation strategy with respect to the optimal relocation frequency under the influence of $c_{p}$.

\subsubsection{The Effect of Arrival Rates}

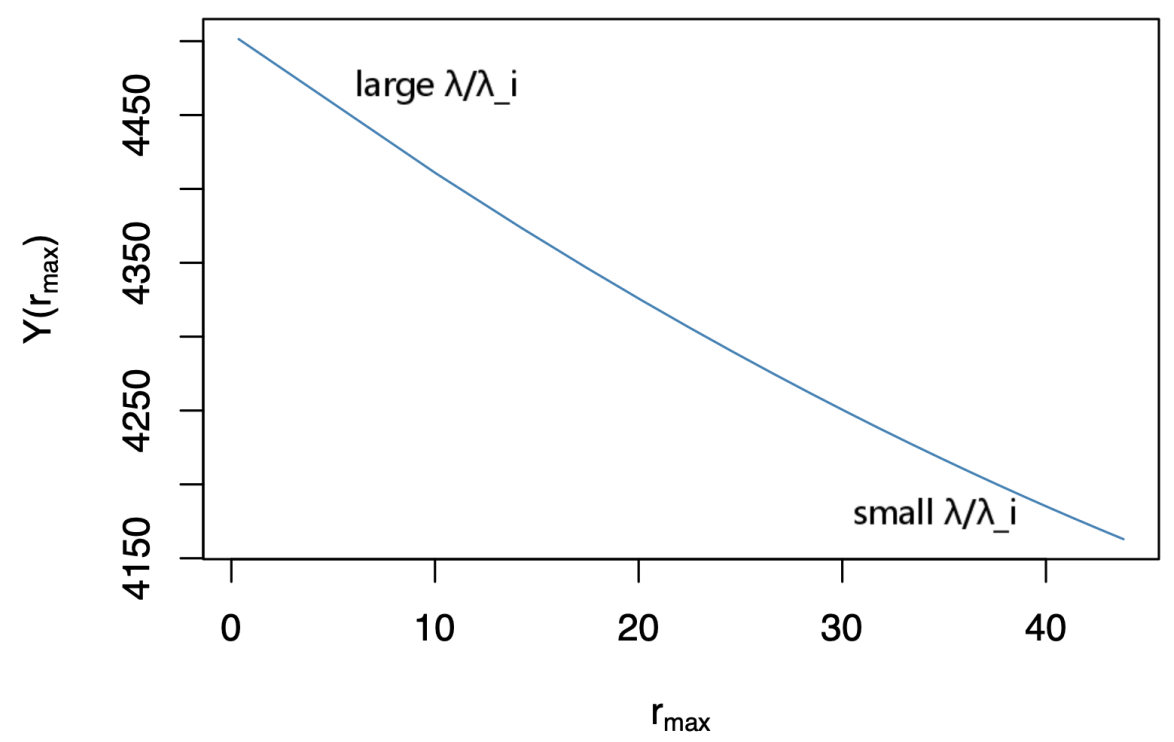

Figure 19. The maximum profit after implementing the relocation strategy with respect to the optimal relocation frequency under the influence of $\frac{\lambda}{\lambda_{i}}$.

The arrival rate is the most important parameter which is concerned with the customer demand for bike service in the paper. The influence of the arrival rates at different nodes on the relocation problem is also investigated by a numerical experiment. In Figure (19), $r_{\max }$ increases and $Y\left(r_{\max }\right)$ decreases with the decrease of $\frac{\lambda}{\lambda_{i}}$. It is because that the decrease of $\frac{\lambda}{\lambda_{i}}$ means the customer demand at different nodes becomes more unequal, which leads to an increased possibility of the imbalanced distribution of bikes in the system. Frequent relocations are needed to deal with this problem. Due to the high cost of frequent relocations, the total profit decreases. From the results, it is observed that compared with the maximum profit, the optimal relocation frequency is more sensitive to the difference of the arrival rates at different nodes in the network, especially when $\frac{\lambda}{\lambda_{i}}$ is small. When $\frac{\lambda}{\lambda_{i}}$ gets close to 1 , the optimal relocation frequency decreases rapidly and becomes very close to 0 . 
Therefore, we suggest that the operators should pay attention to the difference of the customer demand among the nodes, especially when the demand is always changing as the time goes by. Not only does the unequal demand have a significant influence on the bike distribution in the long term, but also it requires multiple times of relocating bikes in the bike-sharing system. If the unit cost of relocations is high, the operators may have to spend a huge amount of money on relocating bikes under the influence of a small value of $\frac{\lambda}{\lambda_{i}}$.

\section{Conclusion}

Compared with the high-demand node, the number of bikes at the lower-demand node changes at a much slower rate. As a whole, we guess that the number of bikes at the higher-demand node is likely to be smaller than the number of bikes at each of the middle-demand nodes (if there are a relatively large number of middle-demand nodes) and is much smaller than the number of bikes at the lower-demand node. The bike distribution at the lower-demand node is not sensitive to the increase of the arrival rate at the higher-demand node, which means bikes at the higher-demand node are likely to be scattered at both the lower-demand node and some middle-demand nodes. It is suggested that the operators should pay more attention to the nodes with higher demands, where the number of bikes decreases rapidly, rather than the nodes with lower demands, where the number of bikes increases relatively slowly, although these all belong to two extremes of the nodes with different customer demand. When the total number of bikes in the system is fixed, the expected number of bikes at the low-demand node in the long run tends to decrease and its possible range becomes broader with the increase of the number of nodes and the decrease of the initial number of bikes at each node. It suggests that a large number of nodes in the network can reduce the influence of the unequal demands moderately and make the long-term bike distribution more balanced than a small number of nodes in the network. Meanwhile, a larger initial number of bikes at each node can lead to a more imbalanced distribution of shared bikes in the system without human intervention.

The relocation problem is solved by an operator-based relocation strategy based on the proper utilization of probabilistic results provided by the theoretical models. The main objective is to maximize the total profit and get the optimal relocation frequency. It is found that the relationship between the total profit and the relocation frequency takes the shape of a concave curve, which makes it quick to find the global optimal point by taking derivative of the expression. There are a variety of key parameters related to the optimization problem (i.e., the indexes which can measure the effectiveness of each relocation $c$ and $\mu$, the unit price $c_{i n}$, the unit cost of relocation $c_{r e}$, the unit cost of unmet demands $c_{p}$ and the different ratios of arrival rates). In our common sense from a business perspective, operators prefer the solution which can bring more profits and requires a low frequency of relocating bikes. The numerical experiments show that small values of $c$ and $c_{p}$ and large values of $\mu$ and can fit the preference. A small value of $c$ and a large value of $\mu$ can reduce the probability of having zero bikes at the high-demand node, which means an effective relocation strategy can result in a low frequency of relocation and increase the total profit mainly by saving the total cost. When the value of $c$ is small, the maximum profit is more sensitive to the change of the optimal relocation frequency, while when the value of $\mu$ is large, the maximum profit is less sensitive to the change of the optimal relocation frequency. Therefore, a small $c$ and a large $\mu$ are complementary to each other, which can provide one of the criteria for operators to select useful relocation strategies. When the ratio of arrival rates is changed in the optimization model, it is indicated that a large difference between the arrival rates is going to decrease the total profit and make the optimal relocation frequency increase rapidly, which totally deviates from the operators' purpose. The unequal demands do not only have an adverse effect on bike distribution, but also influence the relocation frequency and the profit operators can get. What the operators can do is to balance the customer demand among the nodes as much as possible and their efforts spent on balancing can be directly reflected in the benefits. Differing from the influence of $c_{p}$, a large value of $c_{i n}$ and a small value of $c_{r e}$ can increase both the optimal relocation frequency and the maximum total profit, which means the operators can get a maximum profit and also have to 
carry out more frequent relocations. The change of $c_{i n}$ has a greater influence on the total profit and the change of $c_{r e}$ has a greater influence on the optimal relocation frequency. With the decrease of $c_{r e}$, the maximum total profit becomes less sensitive to the change of the optimal relocation frequency, which means the benefit of relocating bikes gradually reduces. Therefore, operators can consider increasing the unit price within a customer's acceptable range in order to make more profits. If the unit cost of each relocation can be reduced significantly, a high frequency of relocation can bring more profits and satisfy more demands. The unit cost of unmet demand is the most uncertain parameter for operators, so it is suggested that the operators should predict the value of $c_{p}$ carefully by investigation and analysis, and minimize the disappointment from customers who cannot find any available bikes to ride if possible.

In short, although our work takes a dockless bike-sharing system as an example, the developed methods and models can be extended to apply in some other resource-sharing systems when necessary. The theoretical models are general, flexible and extensible. By analysis of some factors with practical meanings, this paper can bring real-world insights. There are also some limitations to the study, which can be regarded as directions for further research. The influence of different arrival rates on the distribution of bikes in the dockless bike-sharing system is the focus of our research. In the optimization model for solving the relocation problems, the relocation costs can be estimated based on how many bikes are relocated but do not consider the distance of relocating bikes from one node to another, which is also a limitation of our proposed methods. The optimization models for solving relocation problems can be extended to apply on a more general Markovian network model with multiple high-demand nodes and low-demand nodes in the future study.

\section{References}

1. Stephen D. Parkes and Greg Marsden and Susan A. Shaheen and Adam P. Cohen. Understanding the Diffusion of Public Bikesharing Systems: Evidence from Europe and North America. Journal of Transport Geography 2013, 31, 94-103.

2. Mingshu Wang and Xiaolu Zhou. Bike-sharing systems and congestion: Evidence from US cities. Journal of Transport Geography 2017, 65, 147-154.

3. Yuge Ma and Jing Lan and Thomas Thornton and Diana Mangalagiu and Dajian Zhu. Challenges of collaborative governance in the sharing economy: The case of free-floating bike sharing in Shanghai. Journal of Cleaner Production 2018, 197, 356-365.

4. Nikolaos-Fivos Galatoulas and Konstantinos N. Genikomsakis and Christos S. Ioakimidis. Analysis of potential demand and costs for the business development of an electric vehicle sharing service. Sustainable Cities and Society 2018, 42, 148-161.

5. Inês Frade and Anabela Ribeiro. Bicycle Sharing Systems Demand. Procedia-Social and Behavioral Sciences 2014, 111, 518-527.

6. Chengcheng $\mathrm{Xu}$ and Junyi Ji and Pan Liu. The station-free sharing bike demand forecasting with a deep learning approach and large-scale datasets. Transportation Research Part C: Emerging Technologies 2018, 95, $47-60$.

7. Çelebi, Dilay and Yörüsün, Aslı and Işık, Hanife. Bicycle sharing system design with capacity allocations. Transportation Research Part B: Methodological 2018, 114, 86-98.

8. Maria Bordagaray and Luigi dell'Olio and Achille Fonzone and Ángel Ibeas. Capturing the conditions that introduce systematic variation in bike-sharing travel behavior using data mining techniques. Transportation Research Part C: Emerging Technologies 2016, 71, $231-248$.

9. Krishna Murthy Gurumurthy and Kara M. Kockelman. Analyzing the dynamic ride-sharing potential for shared autonomous vehicle fleets using cellphone data from Orlando, Florida. Computers, Environment and Urban Systems 2018, 71, 177-185.

10. Yuanxuan Yang and Alison Heppenstall and Andy Turner and Alexis Comber. A spatiotemporal and graph-based analysis of dockless bike sharing patterns to understand urban flows over the last mile. Computers, Environment and Urban Systems 2019, 77, 101361. 
11. Yanjie Ji and Xinwei Ma and Mingjia He and Yuchuan Jin and Yufei Yuan. Comparison of usage regularity and its determinants between docked and dockless bike-sharing systems: A case study in Nanjing, China. Journal of Cleaner Production 2020, 255, 120110.

12. Efthimios Bakogiannis and Maria Siti and Stefanos Tsigdinos and Avgi Vassi and Alexandros Nikitas. Monitoring the first dockless bike sharing system in Greece: Understanding user perceptions, usage patterns and adoption barriers. Research in Transportation Business \& Management 2019, 33, 100432.

13. Gyugeun Yoon and Joseph Y.J. Chow. Unlimited-ride bike-share pass pricing revenue management for casual riders using only public data. International Journal of Transportation Science and Technology 2020, 9, 159-169.

14. Arturo Estrada and David Romero and Roberto Pinto and Giuditta Pezzotta and Alexandra Lagorio and Alice Rondini. A Cost-Engineering Method for Product-Service Systems Based on Stochastic Process Modelling: Bergamo's Bike-Sharing PSS. Procedia CIRP 2017, 64, 417-422.

15. Yujing Chen and Yong Zha and Dong Wang and Hongping Li and Gongbing Bi. Optimal pricing strategy of a bike-sharing firm in the presence of customers with convenience perceptions. Journal of Cleaner Production 2020, 253, 119905.

16. Aritra Pal and Yu Zhang. Free-floating bike sharing: Solving real-life large-scale static rebalancing problems. Transportation Research Part C: Emerging Technologies 2020, 80, 92-116.

17. Hamidreza Sayarshad and Sepideh Tavassoli and Fang Zhao. A multi-periodic optimization formulation for bike planning and bike utilization. Transportation Research Part C: Emerging Technologies 2012, 36, 4944-4951.

18. Ying Liu and W.Y. Szeto and Sin C. Ho. A static free-floating bike repositioning problem with multiple heterogeneous vehicles, multiple depots, and multiple visits. Transportation Research Part C: Emerging Technologies 2018, 92, $208-242$.

19. Jan Brinkmann and Marlin W. Ulmer and Dirk C. Mattfeld. Dynamic Lookahead Policies for Stochastic-Dynamic Inventory Routing in Bike Sharing Systems. Computers E Operations Research 2019, 106, 260-279.

20. Benjamin Legros. Dynamic repositioning strategy in a bike-sharing system; how to prioritize and how to rebalance a bike station. European Journal of Operational Research 2019, 272, 740-753.

21. Alfred Benedikt Brendel and Sascha Lichtenberg and Benjamin Brauer and Ilja Nastjuk and Lutz M. Kolbe. Improving electric vehicle utilization in carsharing: A framework and simulation of an e-carsharing vehicle utilization management system. European Journal of Operational Research 2018, 64, 230-245.

22. Svenja Reiss and Klaus Bogenberger. A Relocation Strategy for Munich's Bike Sharing System: Combining an operator-based and a user-based Scheme. Transportation Research Procedia 2017, 22, 104-114.

23. Hamid R. Sayarshad and Joseph Y.J. Chow. Non-myopic relocation of idle mobility-on-demand vehicles as a dynamic location-allocation-queueing problem. Transportation Research Part E: Logistics and Transportation Review 2017, 106, 60-77.

24. Bacem Samet and Florent Couffin and Marc Zolghadri and Maher Barkallah and Mohamed Haddar. Model reduction for studying a Bike Sharing System as a closed queuing network. Procedia Manufacturing 2018, 25, 39-46. 